\title{
Role of thoracic radiotherapy in extensive stage small cell lung cancer: a systemic review and meta-analysis
}

\author{
Ao-Mei Li ${ }^{1 \#}$, Han Zhou ${ }^{1 \#}$, Yang-Yang $\mathrm{Xu}^{2 \#}$, Xiao-Qin $\mathrm{Ji}^{1}$, Tian-Cong $\mathrm{Wu}^{1}$, Xi Yuan ${ }^{1}$, Chang-Chen Jiang ${ }^{1}$, \\ Xi-Xu Zhu', Ping Zhan ${ }^{2,3}$, Ze-Tian Shen ${ }^{1}$ \\ ${ }^{1}$ Department of Radiation Oncology, Jinling Hospital, Medical School of Nanjing University, Nanjing, China; ${ }^{2}$ Department of Respiratory and \\ Critical Care Medicine, Jinling Hospital, Nanjing Medical University, Nanjing, China; ${ }^{3}$ Department of Respiratory and Critical Care Medicine, \\ Jinling Hospital, Nanjing University School of Medicine, Nanjing, China \\ Contributions: (I) Conception and design: P Zhan, ZT Shen; (II) Administrative support: CC Jiang, P Zhan; (III) Provision of study materials or \\ patients: XQ Ji, TC Wu, X Yuan; (IV) Collection and assembly of data: AM Li, H Zhou, YY Xu; (V) Data analysis and interpretation: AM Li, H \\ Zhou, YY Xu; (VI) Manuscript writing: All authors; (VII) Final approval of manuscript: All authors. \\ \#These authors contributed equally to this work. \\ Correspondence to: Ping Zhan. Department of Respiratory and Critical Care Medicine, Jinling Hospital, Nanjing University School of Medicine, \#305, \\ East Zhongshan Road, Nanjing 210002, China. Email: zhanping207@163.com; Ze-Tian Shen. Department of Radiation Oncology, Jinling Hospital, \\ Medical School of Nanjing University, Nanjing, Jiangsu, 210002, China. Email: shen-zetian@163.com.
}

Background: The role of thoracic consolidation radiotherapy in patients with extensive stage small cell lung cancer (ES-SCLC) remains controversial. This study aimed to evaluate the efficacy of thoracic radiotherapy (TRT) in these patients.

Methods: A systematic literature search was performed in PubMed, Embase, and the Cochrane library to identify qualified clinical studies. The hazard ratios (HRs) and 95\% confidence intervals (CIs) of overall survival (OS), progression-free survival (PFS) and local recurrence-free survival (LRFS) were extracted, and toxicity of the TRT group versus non-TRT group was analyzed.

Results: A total of 12 studies were included in this meta-analysis, including 936 patients in the TRT group and 1,059 patients in the non-TRT group. The combined results showed that TRT significantly improved OS (HR =0.65; 95\% CI: 0.55-0.77, P<0.00001), PFS (HR =0.64; 95\% CI: 0.56-0.72, P<0.00001) and LRFS (HR $=0.38,95 \%$ CI: $0.26-0.53, \mathrm{P}<0.00001)$. Subgroup analysis showed that OS benefits were observed in patients receiving sequential TRT ( $\mathrm{HR}=0.67$; 95\% CI: 0.54-0.84, $\mathrm{P}=0.0006)$. The addition of TRT significantly improved $\mathrm{OS}$ in patients over 65 years of age (HR $=0.55$; 95\% CI: 0.40-0.74, $\mathrm{P}=0.0001)$. For patients with only one organ metastasis, there was no significant difference in OS between the two groups (HR $=0.61 ; 95 \%$ CI: $0.36-1.01, \mathrm{P}=0.06$ ). There was no statistical difference in hematologic toxicity (leukopenia, thrombocytopenia, anemia) and non-hematologic toxicity (nausea or vomiting) between the two groups. The incidence of grade $\geq 3$ esophageal toxicity was $4.6 \%$ in the TRT group and $0 \%$ in the non-TRT group $(\mathrm{P}=0.0001)$. Grade $\geq 3$ bronchopulmonary toxicity was $2.9 \%$ in the TRT group and $0.8 \%$ in the non-TRT group $(\mathrm{P}=0.02)$.

Conclusions: TRT improves OS, PFS and LRFS in patients with ES-SCLC, with a low increase in esophageal and bronchopulmonary toxicity. More randomized controlled trials (RCTs) are expected to confirm our conclusions.

PROSPERO registration number: CRD42020190575.

Keywords: Small cell lung cancer (SCLC); thoracic radiotherapy (TRT); meta-analysis

Submitted Aug 08, 2020. Accepted for publication Nov 16, 2020.

doi: $10.21037 /$ atm-20-5765

View this article at: http://dx.doi.org/10.21037/atm-20-5765 


\section{Introduction}

Small cell lung cancer (SCLC) accounts for $10-15 \%$ of all lung cancers. About $70 \%$ of the patients are already in the extensive stage at the initial diagnosis, with a median overall survival (OS) of only $8-10$ months $(1,2)$. So far, platinumbased chemotherapy is still the standard treatment for extensive stage small cell lung cancer (ES-SCLC), with a response rate of up to $60-80 \%$. However, most patients (approximately 90\%) develop intrathoracic progression within 1 year after completion of chemotherapy. There are few effective treatment options, and the 2-year survival rate is only $5 \%(3,4)$.

Since SCLC is sensitive to radiotherapy, many studies have explored the effect of consolidation thoracic radiotherapy (TRT) in ES-SCLC. A randomized controlled trial (RCT) published in 1999 was the first to suggest that the addition of TRT improved OS and first relapse-free survival in ESSCLC. Patients with complete response (CR) at a distant site and at least partial response (PR) at a local site were selected in the study as they had a better prognosis (5). A subsequent RCT demonstrated TRT improved long-term survival in ES-SCLC, with the 2-year OS rate of $13 \%$. In addition, TRT reduced the incidence of intrathoracic progression $(\mathrm{P}<0.0001)(6)$. Recent retrospective analyses and databasebased studies have also demonstrated the survival benefits of thoracic consolidation radiotherapy (7-9). However, the latest randomized trial RTOG-0937 found that despite improved progression-free survival (PFS), the median survival in the TRT group (13.8 months) was worse than that in the prophylactic cranial irradiation (PCI) group (15.8 months) (10). In another retrospective study, no PFS or OS benefit of TRT was observed in patients with brain/ liver/multi-metastasis (11). Therefore, the role of thoracic consolidation radiotherapy in patients with ES-SCLC remains controversial.

In this meta-analysis, we compared the effects of thoracic consolidation radiotherapy versus chemotherapy alone on survival of patients with ES-SCLC by systematically reviewing RCTs and retrospective studies.

We present the following article in accordance with the PRISMA reporting checklist (available at http://dx.doi. org/10.21037/atm-20-5765).

\section{Methods}

\section{Search strategy}

The review was registered in PROSPERO with the registration number CRD42020190575. PubMed, Embase, and the Cochrane library were systematically searched without any language restriction. All electronic database resources are available from the Nanjing Medical University Library. Our team has mastered the search strategy of electronic databases under the guidance of a medical librarian. Search terms were: ("extensive" or "advanced" or "metastatic" or "stage IV") and ("radiation" or "radiotherapy") and ("small cell lung cancer" or "small cell lung carcinoma" or "SCLC"). The last search deadline was July 13, 2020. When necessary, the references quoted in the article were manually retrieved.

\section{Inclusion and exclusion criteria}

The studies which compared the clinical efficacy of TRT combined with chemotherapy and chemotherapy alone for ES-SCLC were eligible for inclusion. Studies that met the following criteria were excluded: (I) insufficient survival data; (II) not ES-SCLC; (III) review, comments, survey, conference abstract, meta-analysis; (IV) not platinum-based chemotherapy as a control group; (V) not TRT.

\section{Data extraction}

Two authors (AML and YYX) independently screened eligible studies and extracted the data. Disagreements were resolved by consulting the third author (HZ). The following information was extracted: author, year of publication, country, study period, number of participants, study type, treatment regimen (including chemotherapy, TRT and PCI), and survival data. We evaluated OS, PFS, local recurrence-free survival (LRFS) and toxicity in the meta-analysis. If both univariate and multivariate analyses of survival data were provided in the study, multivariate analysis results were applied because they reduced confounding bias. Similarly, if survival data both before and after propensity score matching (PSM) were presented in the article, the results after PSM were used.

\section{Quality assessment and statistical analysis}

The quality of retrospective studies was evaluated using the Newcastle-Ottawa Scale (NOS). The possible NOS score of each article ranged from 0 to 9 , and studies with a score greater than 6 were considered to be of high quality (12). The quality of RCTs was assessed using the Cochrane risk of bias tool (13). Two authors (AML and HZ) independently 

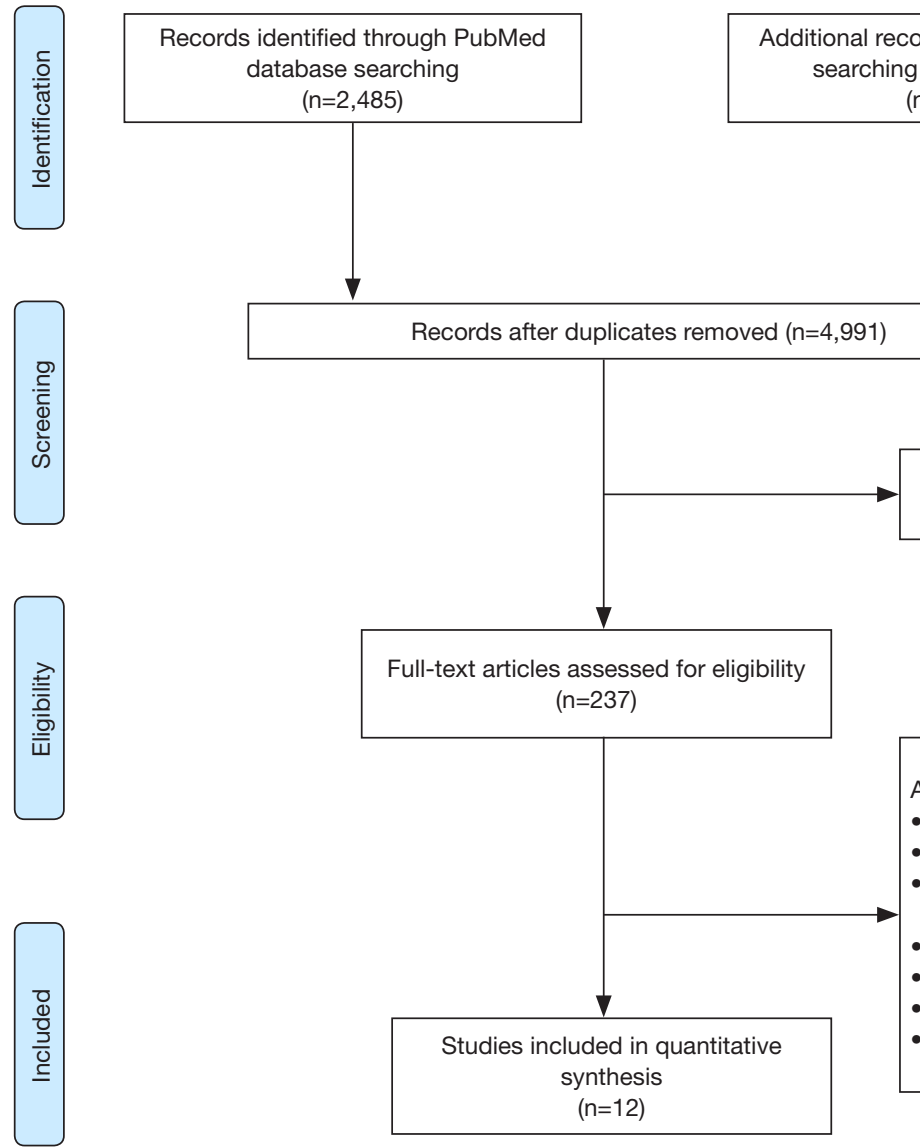

Records excluded because of ineligibility $(n=4,754)$

Articles excluded $(n=225)$

- 9 Insufficient survival data

- 6 Single-arm trial

- 100 Review, comments, survey, conference abstract, metaanalysis

44 Not platinum-based chemotherapy as a control group

- 7 Not thoracic radiotherapy

- 50 Not ES-SCLC

- 9 Unknown chemotherapy regimen

Figure 1 Flow diagram of study selection procedure.

evaluated the included studies. Any inconsistency was resolved by consensus.

Review Manager version 5.3 (Copenhagen: The Nordic Cochrane Centre, The Cochrane Collaboration) was used for statistical analysis. A $\mathrm{P}<0.05$ was considered statistically significant. The effects of thoracic consolidation radiation on OS, PFS and LRFS in patients with ES-SCLC were evaluated by hazard ratios (HRs) and the corresponding 95\% confidence interval (CIs). If the HRs and 95\% CIs were not directly reported in the included article, we used Engauge Digitizer version 11.1 (http://sourceforge.net/ projects/digitizer/) to estimate them from the Kaplan-Meier curve $(14,15)$. Risk ratio (RR) was used to analyze toxicity. Heterogeneity was tested by Cochrane's Q test and I-square $\left(\mathrm{I}^{2}\right)$. The random-effects model was used when significant heterogeneity was observed $\left(\mathrm{P}<0.1\right.$ or $\left.\mathrm{I}^{2}>50 \%\right)$; otherwise, the fixed-effects model was applied. Potential publication bias was assessed by funnel plots. Sensitivity analysis was performed when necessary to explore possible sources of heterogeneity.

\section{Results}

\section{Study selection and characteristics}

We initially retrieved 6,256 studies from the electronic databases, of which 1,265 were duplicates. A total of 4,754 studies were excluded by screening titles and abstracts. After reading the full text of the remaining 237 studies, 12 studies were eventually included in the meta-analysis: 3 RCTs and 9 retrospective studies $(5-6,8-11,16-21)$. The selection process of the study is shown in Figure 1.

A total of 1,995 patients with ES-SCLC were included in this meta-analysis, of whom 936 received thoracic consolidation radiotherapy (TRT group) and 1,059 received chemotherapy alone (non-TRT group). The basic characteristics of the included studies are shown in Table 1. Four retrospective studies performed PSM (8,16-18). Two retrospective studies evaluated the role of TRT in 


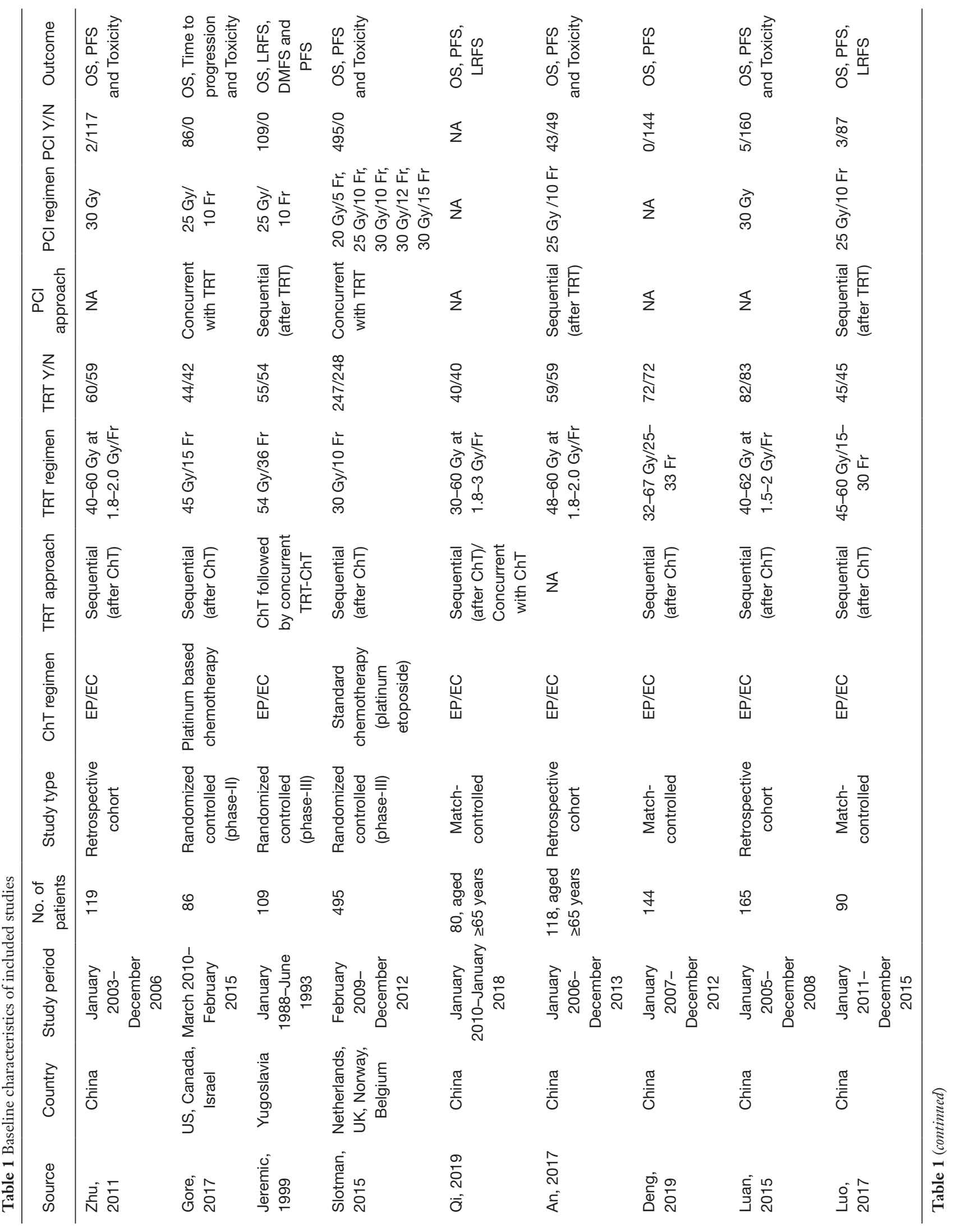




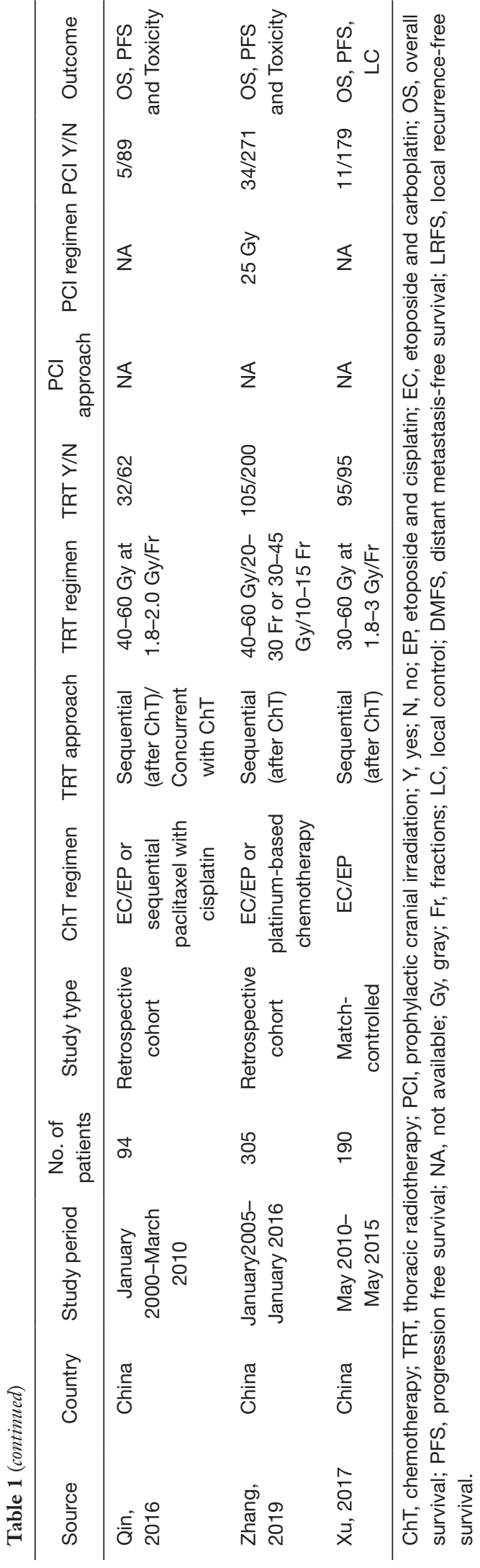

patients aged $\geq 65$ years $(16,19)$. One retrospective study analyzed the prognosis of patients with oligometastases and polymetastases separately (18).

\section{Quality assessment}

All the retrospective studies scored $\geq 7$, and were considered to have high quality. As shown in Table 2, all three RCTs claimed randomization, but no methods for random assignment sequence generation and allocation concealment were reported. The age distribution of patients in the experimental group and the control group in trial RTOG0937 was unbalanced (10).

\section{OS}

All twelve studies reported OS results. Combined analysis showed that TRT significantly improved OS $(\mathrm{HR}=0.65$; 95\% CI: $0.55-0.77, \mathrm{P}<0.00001$ ) (Figure $2 A$ ). As significant heterogeneity was observed $\left(\mathrm{I}^{2}=62 \%\right)$, the random-effects model was applied.

\section{PFS}

All twelve studies reported PFS results. The pooled HR with a random effects model was 0.64 (95\% CI: 0.56-0.72, $\mathrm{P}<0.00001$ ) (Figure $2 B$ ), which indicated that the TRT group had significantly better PFS than non-TRT group.

\section{LRFS}

Four studies compared LRFS between TRT and non-TRT groups. The combined analysis with a random effects model showed that TRT significantly improved LRFS (HR $=0.38$; 95\% CI: 0.26-0.53, $\mathrm{P}<0.00001$ ) (Figure 2C).

\section{Subgroup analysis}

We performed subgroup analysis based on study type to assess the effect of TRT on OS and PFS. The combined HRs for OS of three RCTs and nine retrospective studies were 0.88 (95\% CI: $0.65-1.18, \mathrm{P}=0.39)$ and $0.59(95 \%$ CI: $0.49-0.71, \mathrm{P}<0.00001$ ), respectively (Figure $3 A$ ). The random-effects model was applied as significant heterogeneity was observed ( $\mathrm{I}^{2}=54 \%$ for RCTs). The pooled HR for PFS of three RCTs was 0.71 (95\% CI: 0.61-0.82, $\mathrm{P}<0.00001$ ), and it was 0.61 (95\% CI: 0.52-0.73, $\mathrm{P}<0.00001$ ) for retrospective studies (Figure $3 B$ ). The random-effects 
Table 2 Quality assessment of included studies

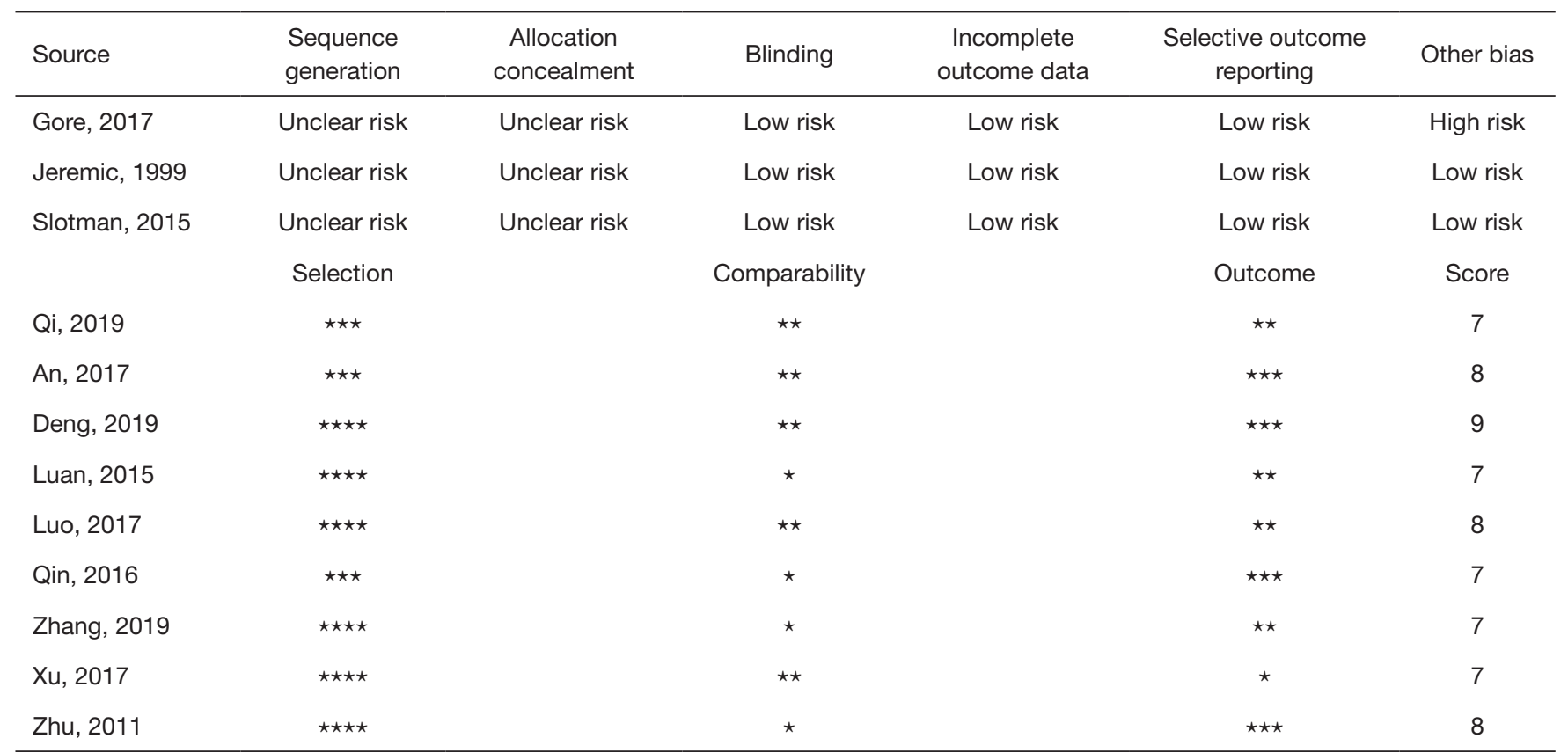

$\star$, one point. Randomized trials were assessed by the Cochrane risk of bias tool. Retrospective studies were assessed by NOS method. A study can be awarded a maximum of one star for each numbered item within the Selection and Outcome categories. A maximum of two stars can be given for Comparability, according to the instruction of NOS.

model was applied as $\mathrm{I}^{2}$ of the pooled retrospective studies was $57 \%$.

\section{Comparison of OS between the TRT and non-TRT groups according to clinicopathologic features}

Nine studies reported OS in patients receiving sequential TRT in combination with platinum-based chemotherapy. The pooled analysis using a random effects model showed that the sequential TRT group had better OS than the non-TRT group (HR $=0.67 ; 95 \%$ CI: $0.54-0.84$, $\mathrm{P}=0.0006$ ) (Figure $4 A$ ). For patients aged $\geq 65$ years, the TRT group had better OS than the non-TRT group (HR $=0.55 ; 95 \%$ CI: $0.40-0.74, \mathrm{P}=0.0001)$. The fixed effects model was used as $\mathrm{I}^{2}=0 \%$ (Figure $4 B$ ). Three studies reported OS in patients with oligometastatic disease (defined as only one organ metastasis). The pooled HR with a random effects model was 0.61 (95\% CI: 0.36-1.01, $\mathrm{P}=0.06$ ) (Figure 4C).

\section{Toxicity}

Grade III or higher toxicity was analyzed in 6 studies, including 570 cases in the TRT group and 694 cases in the non-TRT group. The pooled results with a fixed effects model indicated the TRT group had a higher risk of grade III or higher toxicity than the non-TRT group ( $\mathrm{RR}=1.34$; 95\% CI: $1.17-1.53, \mathrm{P}<0.0001$ ) (Figure 5). Table 3 shows the comparison of common grade III or higher toxicity between the TRT group and the non-TRT group. The pooled RRs were 1.26 (95\% CI: $0.82-1.95, \mathrm{P}=0.29$ ) for grade $\geq 3$ leucopenia, 0.67 (95\% CI: 0.44-1.03, $\mathrm{P}=0.07$ ) for grade $\geq 3$ thrombocytopenia, 0.92 (95\% CI: $0.53-1.59, \mathrm{P}=0.76$ ) for grade $\geq 3$ anemia, 0.61 (95\% CI: $0.35-1.08, \mathrm{P}=0.09$ ) for grade $\geq 3$ nausea or vomiting, 13.89 (95\% CI: 3.63-53.19, $\mathrm{P}=0.0001)$ for grade $\geq 3$ esophageal toxicity and $2.63(95 \%$ CI: $1.21-5.72, \mathrm{P}=0.02$ ) for grade $\geq 3$ bronchopulmonary toxicity.

\section{Sensitivity analysis and publication bias}

The funnel plot was applied to test publication bias. No substantial publication bias was observed with respect to OS (Figure 6). We also performed sensitivity analysis by deleting one study at a time. The results showed that the effect of TRT on OS, PFS and LRFS was reliable. 
A

\begin{tabular}{|c|c|c|c|c|}
\hline Sto & g[Hazard Ratio] & \multicolumn{3}{|c|}{ SE Weight IV.Random. $95 \%$ C } \\
\hline An, 2017 & -0.5155 & 0.2007 & $7.8 \%$ & $0.60[0.40,0.89$ \\
\hline Deng, 2019 & -0.6962 & 0.1804 & $8.5 \%$ & $0.50[0.35,0$. \\
\hline Gore, 2017 & 0.3649 & 0.2874 & $5.5 \%$ & $1.44[0.82,2$. \\
\hline Jeremic, 1999 & -0.3468 & 0.187 & $8.3 \%$ & $0.71[0.49,1.0$ \\
\hline Luan, 2015 & -0.5001 & 0.1899 & $8.2 \%$ & $0.61[0.42,0$. \\
\hline Luo, 2017 & -0.7973 & 0.2248 & $7.1 \%$ & $0.45[0.29,0.70$ \\
\hline Qi, 2019 & -0.7293 & 0.2473 & $6.5 \%$ & $0.48[0.30,0.7$ \\
\hline Qin, 2016 & -0.564 & 0.2567 & $6.2 \%$ & $0.57[0.34$ \\
\hline Slotman, 2015 & -0.1769 & 0.0961 & $11.5 \%$ & $0.84[0.69,1$ \\
\hline$X u, 2017$ & -1.0572 & 0.3647 & $4.0 \%$ & $0.35[0.17,0$. \\
\hline$X u, 2017$ & -0.5151 & 0.1678 & $8.9 \%$ & $0.60[0.43,0$. \\
\hline Zhang, 2019 & 0.0115 & 0.1506 & $9.6 \%$ & $1.01[0.75,1$ \\
\hline Zhu, 2011 & -0.4182 & 0.1952 & $8.0 \%$ & $0.66[0.45,0$. \\
\hline Total $(95 \% \mathrm{Cl})$ & & & $100.0 \%$ & $0.65[0.55,0$. \\
\hline Heterogeneity: & 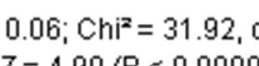 & $=1$ & $=0.00$ & $62 \%$ \\
\hline
\end{tabular}

Hazard Ratio Hazard Ratio

IV. Random. 95\% Cl

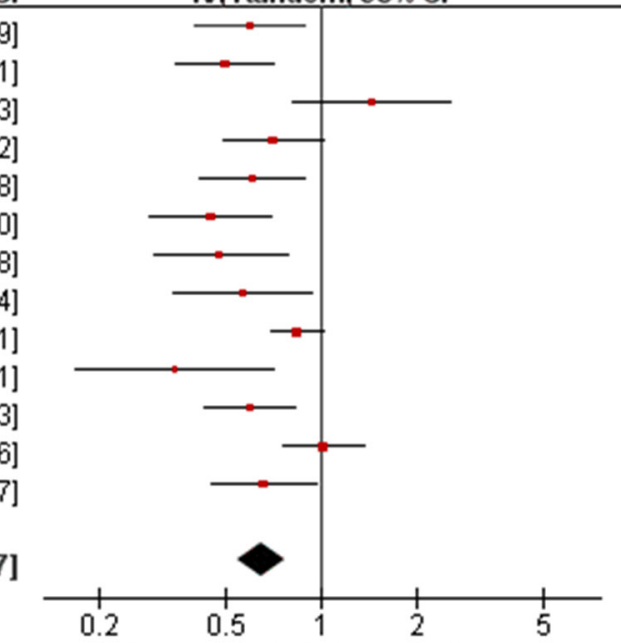

Favours [experimental] Favours [control]

B

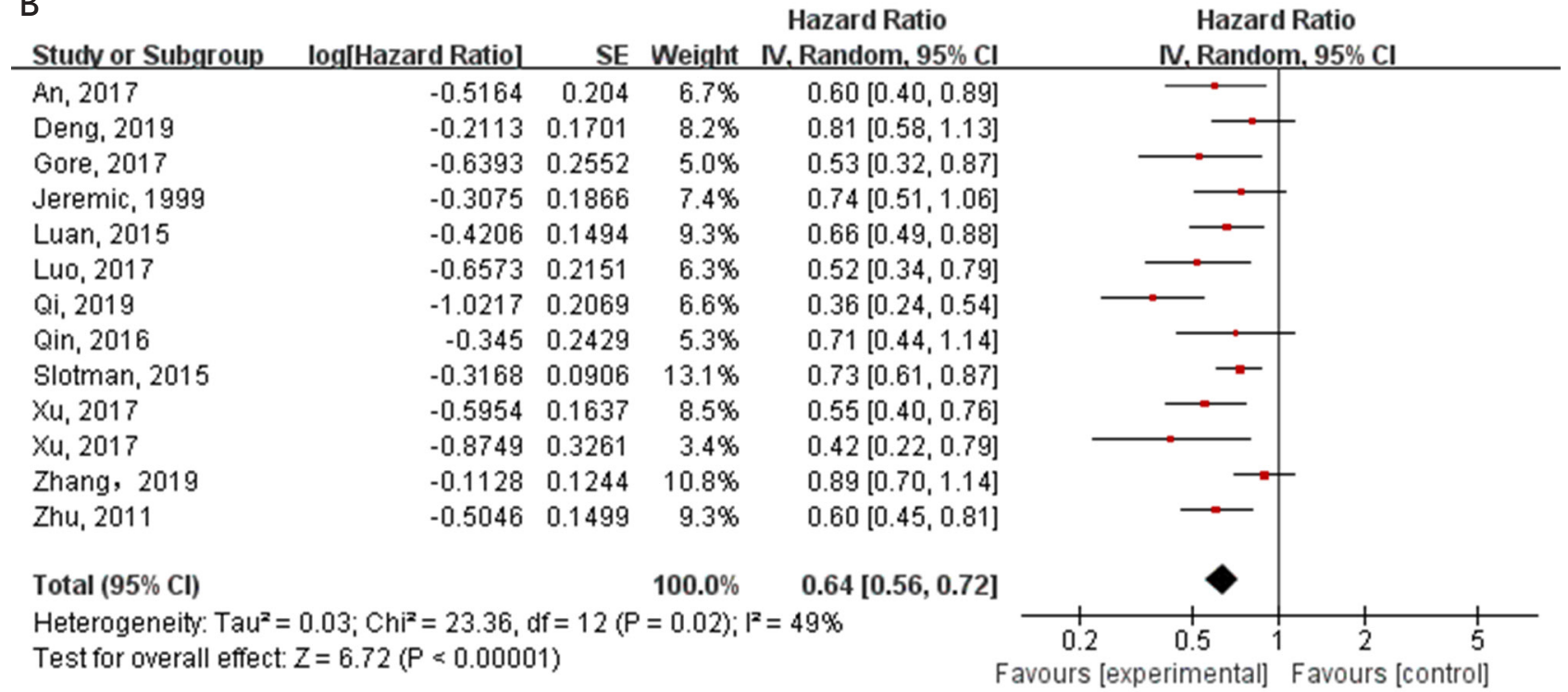

C

\begin{tabular}{|c|c|c|c|c|}
\hline Study or Subgroup & log[Hazard Ratio] & & Weight & Random. $95 \% \mathrm{C}$ \\
\hline Jeremic, 1999 & -0.4734 & 0.226 & $23.2 \%$ & $0.62[0.40,0.97]$ \\
\hline Luo, 2017 & -1.1473 & 0.2895 & $18.8 \%$ & $0.32[0.18,0.56]$ \\
\hline Qi, 2019 & -0.9526 & 0.2421 & $22.0 \%$ & $0.39[0.24,0.62]$ \\
\hline$X u, 2017$ & -1.4001 & 0.2207 & $23.6 \%$ & $0.25[0.16,0.38$ \\
\hline $\mathrm{Xu}, 2017$ & -0.9046 & 0.4134 & $12.4 \%$ & $0.40[0.18,0.91]$ \\
\hline Total $(95 \% \mathrm{Cl})$ & & & $100.0 \%$ & $0.38[0.26,0.5$ \\
\hline
\end{tabular}

Hazard Ratio Hazard Ratio

IV. Random. $95 \% \mathrm{Cl}$

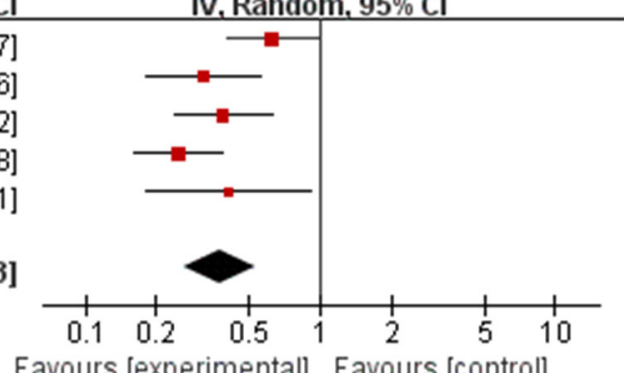

Figure 2 Forest plots of HRs for OS, PFS and LRFS in patients with ES-SCLC. (A) OS; (B) PFS; (C) LRFS. OS, overall survival; PFS, progression-free survival; LRFS, local recurrence-free survival; ES-SCLC, extensive stage small cell lung cancer. 
A

\begin{tabular}{|c|c|c|c|c|}
\hline \multirow{2}{*}{ Studv or Subgroup } & log[Hazard Ratio] & \multicolumn{3}{|r|}{$\mathrm{Cl}$} \\
\hline & \multicolumn{4}{|c|}{ 2.1.1 RCT } \\
\hline Gore, 2017 & 0.3649 & 0.2874 & $19.1 \%$ & $1.44[0.82,2.53]$ \\
\hline Jeremic, 1999 & -0.3468 & 0.187 & $31.8 \%$ & $0.71[0.49,1.02]$ \\
\hline Slotman, 2015 & -0.1806 & 0.0972 & $49.1 \%$ & $0.83[0.69,1.01]$ \\
\hline Subtotal (95\% Cl) & & & $100.0 \%$ & $0.88[0.65,1.18]$ \\
\hline \multicolumn{5}{|c|}{ Heterogeneity: $\mathrm{Tau}^{2}=0.04 ; \mathrm{Chi}^{2}=4.37, \mathrm{df}=2(\mathrm{P}=0.11) ; \mathrm{I}^{2}=54 \%$} \\
\hline \multicolumn{5}{|l|}{ 2.1.2 non-RCT } \\
\hline An, 2017 & -0.5155 & 0.2007 & $10.5 \%$ & $0.60[0.40,0.89]$ \\
\hline Deng, 2019 & -0.6962 & 0.1804 & $11.6 \%$ & $0.50[0.35,0.71]$ \\
\hline Luan, 2015 & -0.5001 & 0.1899 & $11.0 \%$ & $0.61[0.42,0.88]$ \\
\hline Luo, 2017 & -0.7973 & 0.2248 & $9.3 \%$ & $0.45[0.29,0.70]$ \\
\hline Qi, 2019 & -0.7293 & 0.2473 & $8.3 \%$ & $0.48[0.30,0.78]$ \\
\hline Qin, 2016 & -0.564 & 0.2567 & $8.0 \%$ & $0.57[0.34,0.94]$ \\
\hline$x u, 2017$ & -1.0572 & 0.3647 & $4.9 \%$ & $0.35[0.17,0.71]$ \\
\hline$x u, 2017$ & -0.5151 & 0.1678 & $12.3 \%$ & $0.60[0.43,0.83]$ \\
\hline Zhang, 2019 & 0.0115 & 0.1506 & $13.4 \%$ & $1.01[0.75,1.36]$ \\
\hline Zhu, 2011 & -0.4182 & 0.1952 & $10.8 \%$ & $0.66[0.45,0.97]$ \\
\hline Subtotal $(95 \% \mathrm{Cl})$ & & & $100.0 \%$ & $0.59[0.49,0.71]$ \\
\hline \multicolumn{5}{|c|}{ Heterogeneity: $\operatorname{Tau}^{2}=0.04 ; \mathrm{Chi}^{2}=17.88, \mathrm{df}=9(\mathrm{P}=0.04) ; \mathrm{I}^{2}=50 \%$} \\
\hline
\end{tabular}

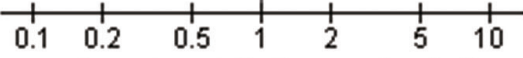

Favours [experimental] Favours [control]

Test for subaroun differences: $\mathrm{Chi}^{2}=5.07 . \mathrm{df}=1(\mathrm{P}=0.02) . \mathrm{I}^{\mathrm{2}}=80.3 \%$

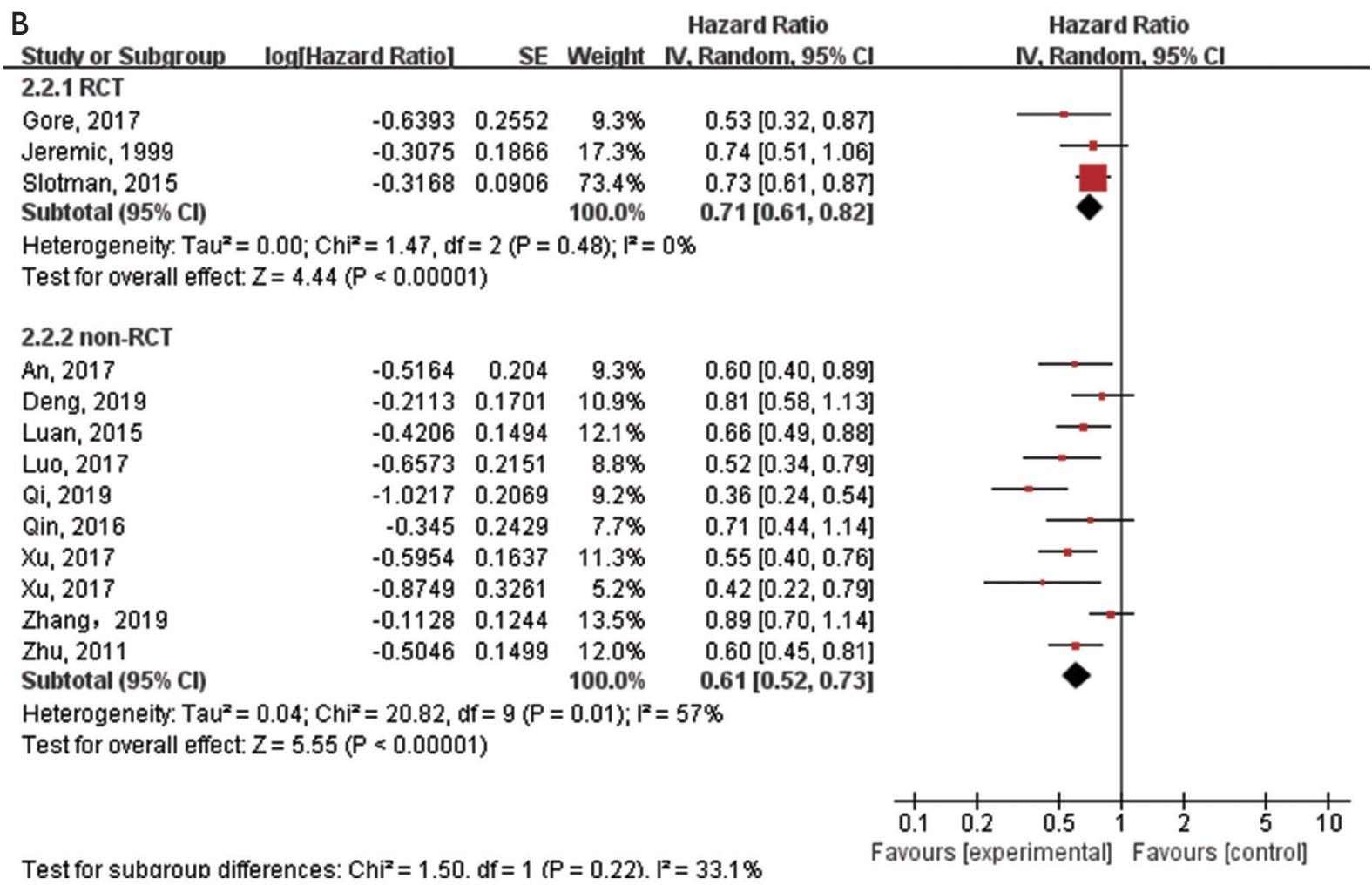

Figure 3 Forest plots for subgroup analysis of HRs for OS and PFS by study type. (A) OS; (B) PFS. OS, overall survival; PFS, progressionfree survival. 
A

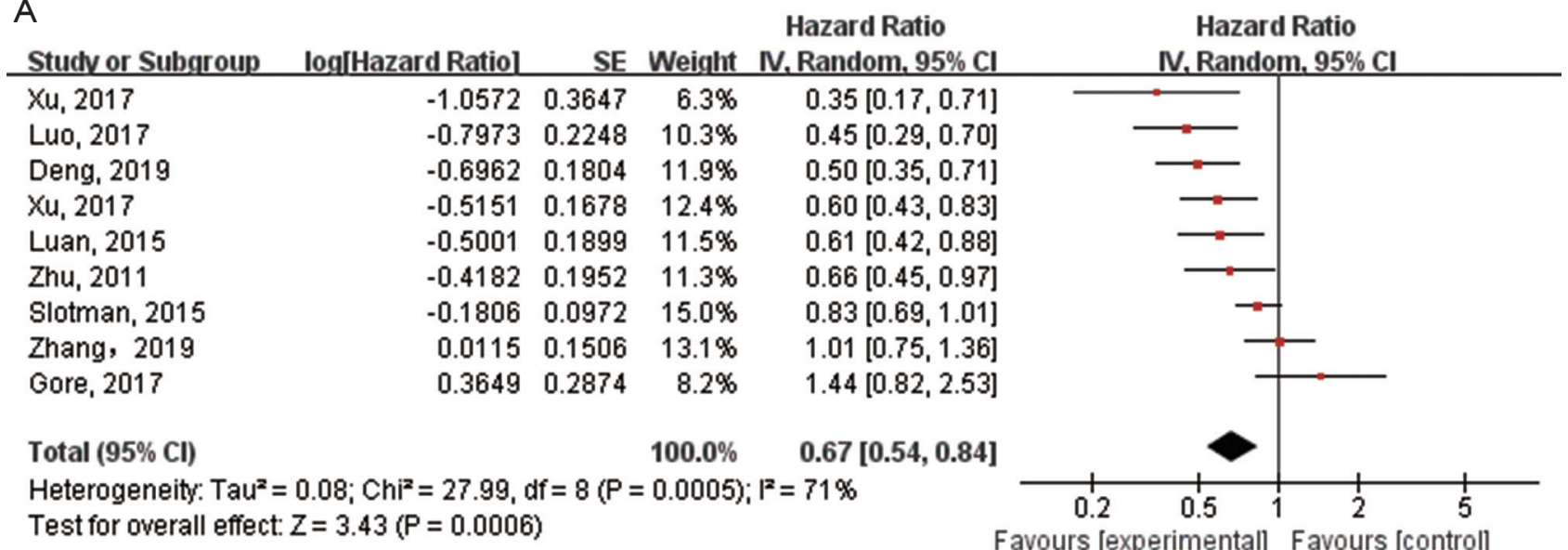

B

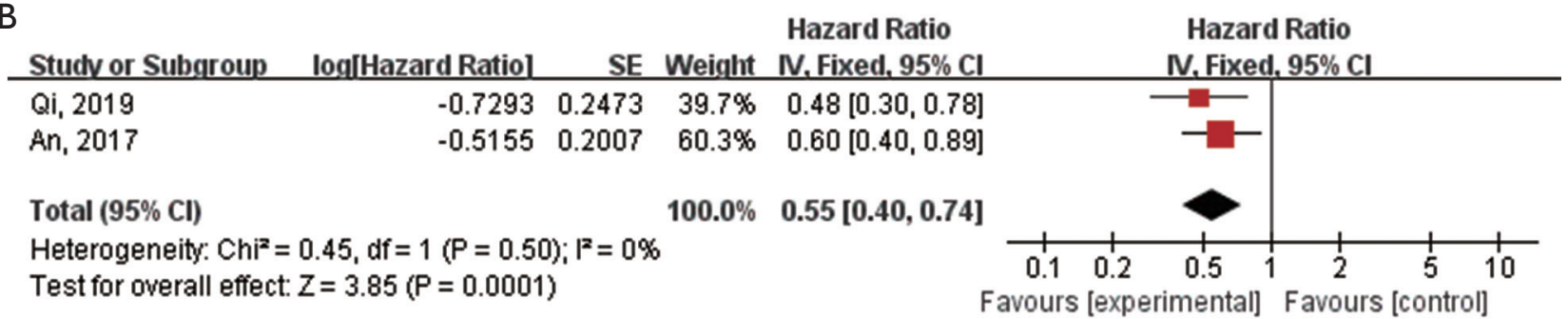

C

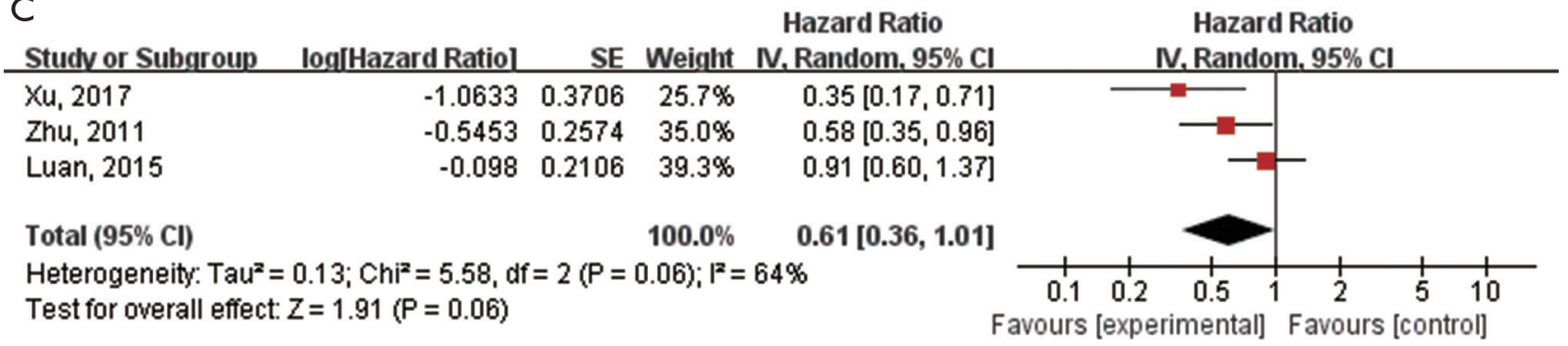

Figure 4 Forest plots of HRs for OS in patients with different clinicopathological features. (A) Patients receiving sequential TRT; (B) patients over 65 years old; (C) patients with oligometastatic disease. OS, overall survival.

\section{Discussion}

This meta-analysis compared the clinical efficacy of TRT combined with chemotherapy with chemotherapy alone in ES-SCLC based on 12 studies. The combined results showed that TRT significantly improved both OS and PFS. In addition, TRT reduced the risk of intrathoracic progression and improved LRFS.

Subgroup analysis according to the study type was conducted due to significant heterogeneity of the combined results. The benefits of TRT on OS and PFS were observed in the subgroup of retrospective studies. In the subgroup of RCTs, TRT still improved PFS in patients with ES-SCLC.
However, the pooled analysis of RCTs showed that there was no significant difference in OS between the two groups, which was consistent with the previous meta-analysis (including the same three RCTs) (22). Moderate degree of heterogeneity was observed in the RCT subgroup, which was mainly affected by the negative results of the RTOG0937 (10). Baseline characteristics of the TRT group and the non-TRT group in RTOG-0937 were unbalanced, and there were more elderly patients ( 65 years or older) in the TRT group, which may increase the risk of bias. In addition, the TRT group included more patients with poor performance status, 2-4 metastatic lesions, and PR to initial 


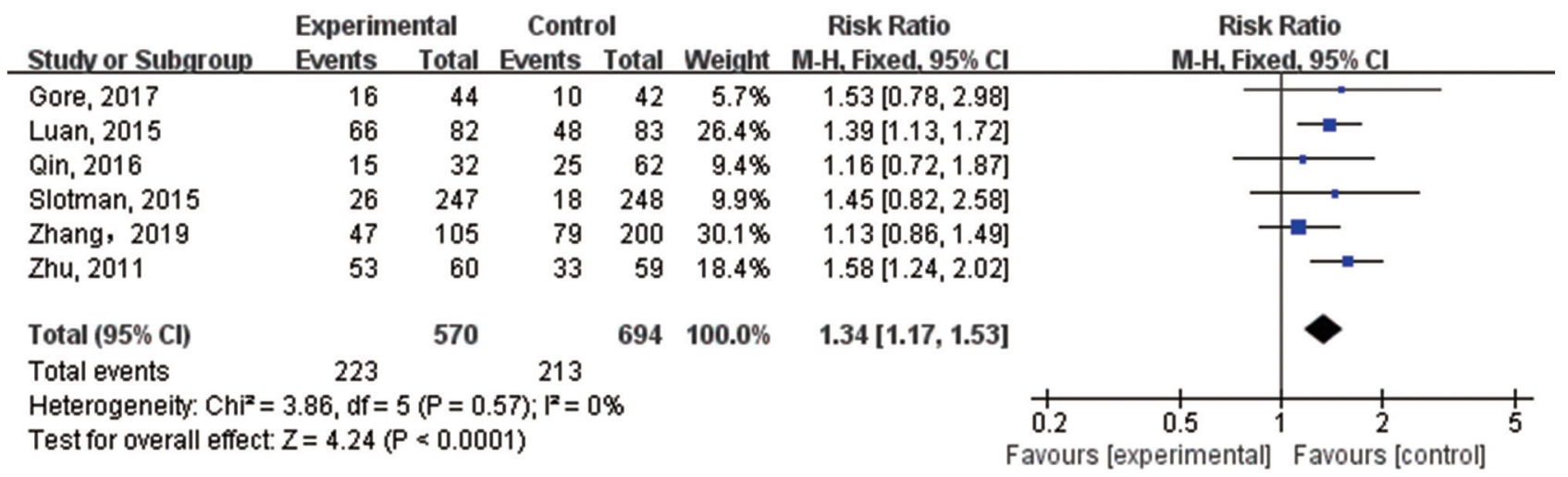

Figure 5 Forest plot of RR for grade III or higher toxicity in patients with ES-SCLC. ES-SCLC, extensive stage small cell lung cancer.

Table 3 Comparison of common grade III or higher toxicity between the TRT group and the non-TRT group

\begin{tabular}{|c|c|c|c|c|c|c|c|c|c|c|}
\hline Toxicity (grade $\geq 3$ ) & $\begin{array}{l}\text { No. of } \\
\text { studies }\end{array}$ & \multicolumn{3}{|c|}{ TRT group } & \multicolumn{3}{|c|}{ Non-TRT group } & $\mathrm{RR}(95 \% \mathrm{Cl})$ & $\begin{array}{l}\text { Heterogeneity } \\
\qquad\left(I^{2}\right)\end{array}$ & $P$ value \\
\hline Leucopenia & 5 & 116 & 273 & 42.5 & 98 & 300 & 32.7 & $1.26(0.82-1.95)$ & $70 \%$ & 0.29 \\
\hline Thrombocytopenia & 5 & 27 & 273 & 9.9 & 42 & 300 & 14 & $0.67(0.44-1.03)$ & $0 \%$ & 0.07 \\
\hline Anemia & 5 & 22 & 273 & 8.1 & 25 & 300 & 8.3 & $0.92(0.53-1.59)$ & $0 \%$ & 0.76 \\
\hline Esophageal toxicity & 6 & 27 & 581 & 4.6 & 0 & 716 & 0 & 13.89 (3.63-53.19) & $0 \%$ & 0.0001 \\
\hline $\begin{array}{l}\text { Bronchopulmonary } \\
\text { toxicity }\end{array}$ & 7 & 18 & 625 & 2.9 & 6 & 748 & 0.8 & $2.63(1.21-5.72)$ & $3 \%$ & 0.02 \\
\hline
\end{tabular}

Grade $\geq 3$ esophageal toxicity includes: esophagitis, dysphagia; Grade $\geq 3$ bronchopulmonary toxicity includes: dyspnea, cough, pneumonitis. TRT, thoracic radiotherapy; RR, risk ratio.

chemotherapy, which may further weaken the survival advantage of consolidation radiotherapy.

Considering that the survival benefit of platinumbased chemotherapy and concurrent TRT was superior to sequential TRT in patients with limited-stage SCLC, we explored the role of sequential radiotherapy in ES-SCLC (23). Our analysis suggested that sequential TRT had better OS than the control group. The effect of concurrent TRT in ES-SCLC was not evaluated due to insufficient data. Of the twelve included studies, only one randomized study compared survival in patients receiving concurrent TRT with those receiving chemotherapy alone. The results showed that concurrent TRT provided a significant survival benefit (5).

In the meta-analysis, we further explored the role of TRT in patients with ES-SCLC with different clinicopathologic features. The pooled results showed that the addition of TRT still improved the prognosis of patients over 65 years of age. In addition, previous studies have demonstrated that ES-SCLC patients with a single metastatic site were more suitable for radiotherapy as they had lower disease burden $(16,24,25)$. However, our study did not observe the benefit of TRT for OS in patients with only one organ metastasis. Since only three studies have reported OS in patients with oligometastatic disease and they were all retrospective studies, this conclusion should be considered with caution. The study of Zhang et al. was not included in this subgroup analysis because they excluded patients with brain or liver metastases from the oligometastatic population. In their study, TRT significantly increased PFS and OS in patients with oligometastatic ES-SCLC without brain or liver metastasis (11). It is worth noting that a secondary analysis of the CREST trial found that both OS and PFS benefits were observed in patients with two or fewer metastases (no brain metastasis). No difference in survival was observed between patients with $0-1$ metastatic sites and 2 metastatic 


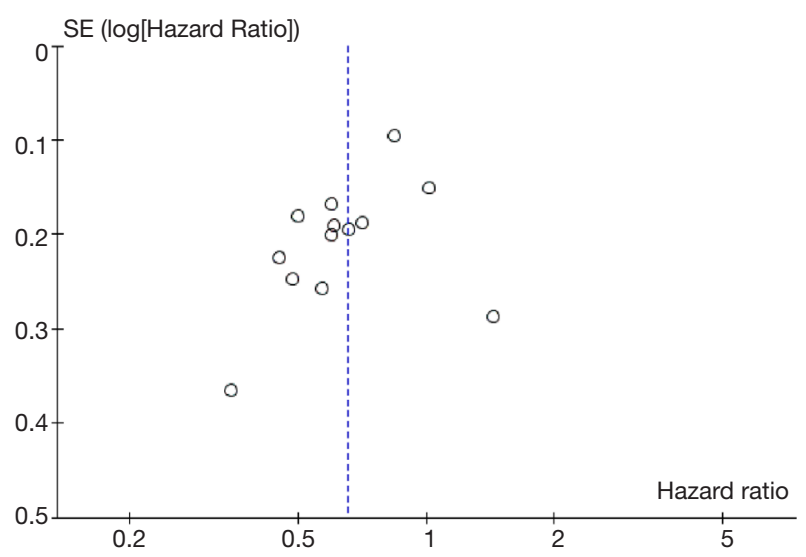

Figure 6 Funnel plot for publication bias.

sites (25). Moreover, in the first randomized controlled study that found the survival benefits of TRT in ES-SCLC, most patients (about $90 \%$ ) had two or fewer metastases (no brain metastasis) (5). Therefore, the definition of oligometastases (with or without brain metastasis, one or two metastasis sites) in future studies still needs to be unified. Because of the limited number of studies, we did not conduct a subgroup analysis of survival in patients with multiple metastases. A recent retrospective study found that TRT also significantly improved prognosis in patients with multiple metastases (18). Another study showed that there was no statistical difference in PFS and OS between TRT and non-TRT groups for patients with brain/liver/multimetastasis (11). In the future, further prospective RCTs are needed to assess the role of TRT in ES-SCLC patients with different numbers of metastatic sites. As far as we know, our study is the first to report these results.

Our meta-analysis suggested that patients receiving consolidation TRT had a higher risk of grade $\geq 3$ toxicity. We then compared the common grade $\geq 3$ toxicity between the TRT group and the control group, and found no statistical difference in hematologic toxicity (leukopenia, thrombocytopenia, anemia) and non-hematologic toxicity (nausea or vomiting) between the two groups. Consolidation TRT resulted in a higher incidence of grade III or higher esophageal and bronchopulmonary toxicity than the nonTRT group. However, the incidence of grade $\geq 3$ esophageal (4.6\%) and bronchopulmonary (2.9\%) toxicity in TRT group was low. Furthermore, radiation-induced deaths were infrequent, with only two treatment-related deaths in all included studies. They all died of radiation pneumonitis $(10,21)$. In conclusion, chemotherapy combined with TRT was well tolerated in patients with ES-SCLC.
There are some limitations in our study. First, the survival data in some studies were estimated from KaplanMeier curves, which may reduce the accuracy of the results. Second, our study included three RCTs and nine retrospective studies. Retrospective studies may introduce selection bias. Third, moderate degree of heterogeneity was found in some combined analyses (all $\left.\mathrm{I}^{2}<75 \%\right)$. This may be due to the differences in baseline characteristics (performance score, brain metastasis, disease burden), chemotherapy-related issues (response to initial chemotherapy, chemotherapy cycle), radiotherapy regimen (radiation dose, radiotherapy timing), and PCI or not in the included study. Subgroup analyses were not conducted due to insufficient information on these factors in the included studies. However, we performed the sensitivity analysis and found that the benefits of TRT on OS, PFS and LRFS were not affected after excluding any single study, which confirmed the stability of our results.

\section{Conclusions}

Our meta-analysis showed that TRT improves OS, PFS and LRFS in patients with ES-SCLC. Further RCTs are expected to confirm our conclusions.

\section{Acknowledgments}

Funding: This work was supported by grants from the National Natural Science Foundation of China (grant numbers 81401903,81572937 and 81572273); the 16th batch "Summit of the Six Top Talents" Program of Jiangsu Province (grant number WSN-154); China Postdoctoral Science Foundation 12th batch Special fund (Postdoctoral number: 45786); China Postdoctoral Science Foundation 64th batch (Postdoctoral number: 45786); Jiangsu Provincial Postdoctoral Science Foundation (grant number 2018K049A); the Natural Science Foundation of Jiangsu province (grant numbers BK20180139 and BK20161386); Jiangsu Provincial Medical Youth Talent (grant number QNRC2016125); the Nanjing Medical Science and Technology Development Project (No. ZKX17044); and the Jiangsu Provincial Key Research and Development Program (No. BE2016721).

\section{Footnote}

Reporting Checklist: The authors have completed the PRISMA reporting checklist. Available at http://dx.doi. 
org/10.21037/atm-20-5765

Conflicts of Interest: All authors have completed the ICMJE uniform disclosure form (available at http://dx.doi. org/10.21037/atm-20-5765). The authors have no conflicts of interest to declare.

Ethical Statement: The authors are accountable for all aspects of the work in ensuring that questions related to the accuracy or integrity of any part of the work are appropriately investigated and resolved.

Open Access Statement: This is an Open Access article distributed in accordance with the Creative Commons Attribution-NonCommercial-NoDerivs 4.0 International License (CC BY-NC-ND 4.0), which permits the noncommercial replication and distribution of the article with the strict proviso that no changes or edits are made and the original work is properly cited (including links to both the formal publication through the relevant DOI and the license). See: https://creativecommons.org/licenses/by-nc-nd/4.0/.

\section{References}

1. Rudin CM, Giaccone G, Ismaila N. Treatment of SmallCell Lung Cancer: American Society of Clinical Oncology Endorsement of the American College of Chest Physicians Guideline. J Oncol Pract 2016;12:83-6.

2. Parikh M, Riess J, Lara PN Jr. New and emerging developments in extensive-stage small cell lung cancer therapeutics. Curr Opin Oncol 2016;28:97-103.

3. Slotman B, Faivre-Finn C, Kramer G, et al. Prophylactic cranial irradiation in extensive small-cell lung cancer. $\mathrm{N}$ Engl J Med 2007;357:664-72.

4. Alvarado-Luna G, Morales-Espinosa D. Treatment for small cell lung cancer, where are we now?-a review. Transl Lung Cancer Res 2016;5:26-38.

5. Jeremic B, Shibamoto Y, Nikolic N, et al. Role of radiation therapy in the combined-modality treatment of patients with extensive disease small-cell lung cancer: A randomized study. J Clin Oncol 1999;17:2092-9.

6. Slotman BJ, van Tinteren H, Praag JO, et al. Use of thoracic radiotherapy for extensive stage small-cell lung cancer: a phase 3 randomised controlled trial. Lancet 2015;385:36-42.

7. Zhang R, Li P, Li Q, et al. Radiotherapy improves the survival of patients with extensive-disease small-cell lung cancer: a propensity score matched analysis of Surveillance,
Epidemiology, and End Results database. Cancer Manag Res 2018;10:6525-35.

8. Deng L, Zhou Z, Xiao Z, et al. Impact of thoracic radiation therapy after chemotherapy on survival in extensive-stage small cell lung cancer: A propensity scorematched analysis. Thorac Cancer 2019;10:799-806.

9. Qin T, Zhou N, Zeng YD, et al. Benefit from thoracic radiotherapy in patients with extensive-disease small-cell lung cancer with elevated lactate dehydrogenase. Onco Targets Ther 2016;9:1095-103.

10. Gore EM, Hu C, Sun AY, et al. Randomized Phase II Study Comparing Prophylactic Cranial Irradiation Alone to Prophylactic Cranial Irradiation and Consolidative Extracranial Irradiation for Extensive-Disease Small Cell Lung Cancer (ED SCLC): NRG Oncology RTOG 0937. J Thorac Oncol 2017;12:1561-70.

11. Zhang H, Deng L, Wang X, et al. Metastatic location of extensive stage small-cell lung cancer: implications for thoracic radiation. J Cancer Res Clin Oncol 2019;145:2605-12.

12. Stang A. Critical evaluation of the Newcastle-Ottawa scale for the assessment of the quality of nonrandomized studies in meta-analyses. Eur J Epidemiol 2010;25:603-5.

13. Higgins JP, Altman DG, Gøtzsche PC, et al. The Cochrane Collaboration's tool for assessing risk of bias in randomised trials. BMJ 2011;343:d5928.

14. Parmar MK, Torri V, Stewart L. Extracting summary statistics to perform meta-analyses of the published literature for survival endpoints. Stat Med 1998;17:2815-34.

15. Tierney JF, Stewart LA, Ghersi D, et al. Practical methods for incorporating summary time-to-event data into metaanalysis. Trials 2007;8:16.

16. Qi J, Xu L, Sun J, et al. Thoracic Radiotherapy Benefits Elderly Extensive-Stage Small Cell Lung Cancer Patients with Distant Metastasis. Cancer Manag Res 2019;11:10767-75.

17. Luo J, Xu L, Zhao L, et al. Timing of thoracic radiotherapy in the treatment of extensive-stage small-cell lung cancer: important or not? Radiat Oncol 2017;12:42.

18. Xu LM, Cheng C, Kang M, et al. Thoracic radiotherapy (TRT) improved survival in both oligo- and polymetastatic extensive stage small cell lung cancer. Sci Rep 2017;7:9255.

19. An C, Jing $W$, Zhang $Y$, et al. Thoracic radiation therapy could give survival benefit to elderly patients with extensive-stage small-cell lung cancer. Future Oncol 2017;13:1149-58.

20. Luan Z, Wang Z, Huang W, et al. Efficacy of 3D 
conformal thoracic radiotherapy for extensive-stage smallcell lung cancer: A retrospective study. Exp Ther Med 2015;10:671-8.

21. Zhu H, Zhou Z, Wang Y, et al. Thoracic radiation therapy improves the overall survival of patients with extensivestage small cell lung cancer with distant metastasis. Cancer 2011;117:5423-31.

22. Rathod S, Jeremic B, Dubey A, et al. Role of thoracic consolidation radiation in extensive stage small cell lung cancer: A systematic review and metaanalysis of randomised controlled trials. Eur J Cancer 2019;110:110-9.

23. Takada M, Fukuoka M, Kawahara M, et al. Phase

Cite this article as: $\mathrm{Li} \mathrm{AM}$, Zhou $\mathrm{H}, \mathrm{Xu} Y Y, \mathrm{Ji} X Q$, Wu TC, Yuan X, Jiang CC, Zhu XX, Zhan P, Shen ZT. Role of thoracic radiotherapy in extensive stage small cell lung cancer: a systemic review and meta-analysis. Ann Transl Med 2021;9(4):299. doi: 10.21037/atm-20-5765
III study of concurrent versus sequential thoracic radiotherapy in combination with cisplatin and etoposide for limited-stage small-cell lung cancer: results of the Japan Clinical Oncology Group Study 9104. J Clin Oncol 2002;20:3054-60.

24. Fukui T, Itabashi M, Ishihara M, et al. Prognostic factors affecting the risk of thoracic progression in extensive-stage small cell lung cancer. BMC Cancer 2016;16:197.

25. Slotman BJ, Faivre-Finn C, van Tinteren H, et al. Which patients with ES-SCLC are most likely to benefit from more aggressive radiotherapy: A secondary analysis of the Phase III CREST trial. Lung Cancer 2017;108:150-3. 